\title{
Ionizing radiation-induced micronucleus formation is mediated by reactive oxygen species that are produced in a manner dependent on mitochondria, Nox1, and JNK
}

\author{
KYUNG-MI CHOI ${ }^{1}$, CHANG-MO KANG ${ }^{2}$, EUN SOOK CHO ${ }^{1}$, SEONG MAN KANG $^{3}$, \\ SEUNG BUM LEE ${ }^{1}$ and HONG-DUCK UM ${ }^{1}$ \\ Laboratories of ${ }^{1}$ Radiation Tumor Physiology, ${ }^{2}$ Radiation Cytogenetics and Epidemiology, \\ Korea Institute of Radiological and Medical Sciences, Seoul 139-706; ${ }^{3}$ Graduate \\ School of Biotechnology, Korea University, Seoul 136-701, Korea
}

Received December 1, 2006; Accepted January 19, 2007

\begin{abstract}
Ionizing radiation (IR) is known to induce genotoxic damage to DNA, chromosomes, and the nucleus. However, the damage that IR causes to the nucleus has received much less attention. Given that reactive oxygen species (ROS) are involved in IR-induced DNA breaks and chromosomal aberrations, this study examined the role of ROS in IR-induced damage to the nucleus. Human Jurkat $\mathrm{T}$ cells were irradiated with $\gamma$-rays at a dose of $2.5 \mathrm{~Gy}$, which resulted in a dramatic increase in both the cellular ROS levels and the number of micronuclei. This latter event was attenuated when the IRinduced ROS were eliminated through the exogenous application of an antioxidant enzyme catalase. The ability of IR to induce the accumulation of ROS and micronucleus formation was also reduced either when the cells were irradiated in the presence of rotenone, a mitochondrial respiratory chain inhibitor, or when the cellular Nox1 levels were reduced by RNA interference. These results suggest that IR stimulates both the mitochondria and Nox 1 to produce ROS, and that these ROS are involved in the IR-induced formation of micronuclei. IR also activated c-Jun N-terminal kinase (JNK), which was reversed by catalase, rotenone, or Nox1 RNA interference. SP600125, a JNK-specific inhibitor, suppressed the IR-induced accumulation of ROS. This inhibitor consistently attenuated the IR-induced formation of micronuclei. Therefore, ROS and JNK appear to act in a positive mutual manner in IR-induced signaling processes. Overall, IR appears to induce the formation of micronuclei by inducing ROS through mitochondria, Nox1, and JNK.
\end{abstract}

Correspondence to: Dr Hong-Duck Um, Laboratory of Radiation Tumor Physiology, Korea Institute of Radiological and Medical Sciences, 215-4 Gongneung-dong, Nowon-gu, Seoul 139-706, Korea E-mail: hdum@kcch.re.kr

Key words: cell signaling, genotoxic damage, ionizing radiation, c-Jun $\mathrm{N}$-terminal kinase, micronucleus, reactive oxygen species

\section{Introduction}

Ionizing radiation (IR) is a genotoxic agent that is widely used in cancer treatment. IR-induced genotoxic damage has been observed in DNA, chromosomes, and nucleus. Examples of these include DNA breaks, chromosomal aberrations, and micronuclei formation (1-3). While the mechanisms underlying these effects are not completely understood, it is generally accepted that IR can induce DNA breakage either by directly ionizing DNA or by generating reactive oxygen species (ROS) $(4,5)$. The role of ROS is supported by the observation that the exogenous application of antioxidants attenuated the ability of IR to induce DNA damage under a variety of experimental conditions $(6,7)$. Since antioxidants have similar protective effects against IR-induced chromosomal aberrations (8), it appears that ROS are commonly involved in both the DNA and chromosomal damage induced by IR. However, it is unclear if ROS can also mediate the IR-induced nuclei damage.

The cellular ROS levels can be increased in response to many types of external stimuli, including growth factors, cytokines, and environmental stress (9-12). As the induced ROS can act as signaling messengers that regulate various cell functions such as growth, differentiation, and viability, many studies have focused on the cellular processes leading to the induction of ROS. Consequently, the mitochondria (13-15) and the Nox family of NADPH oxidase (16-18) have become major sources of ROS induced by external stimuli. The mitochondria generate ROS as byproducts of respiration, and rotenone, an inhibitor of the mitochondrial respiratory chain, has been shown to attenuate the ROS induction under diverse experimental conditions (13-15). NADPH oxidase is a membrane enzyme that is responsible for the oxidative burst induced in activated phagocytes $(17,18)$. Recent studies have suggested that nonphagocytic cells can express the isoforms of the catalytic subunit (Nox2) of NADPH oxidase, such as Nox1, Nox3, Nox4, and Nox5 (19-21). Among these, Nox1 has been relatively well characterized, and shown to be involved in the growth factor-induced ROS production in smooth muscle and epithelial cells (22).

Given the role of the mitochondria and Nox isozymes in the induction of ROS, IR may induce the production of ROS 
by triggering their actions. It was indeed reported that rotenone could prevent the IR-induced accumulation of ROS (23), which suggests the involvement of the mitochondria in the generation of IR-induced ROS. Hence, it is possible that the mitochondria contribute to the genotoxic action of IR. It is also possible that Nox isozymes respond to IR to induce the generation of ROS with the resulting genotoxic effects. However, these possibilities have not been directly addressed, even though this information is essential for understanding the actions of IR and for the development of more efficient and safer strategies for the therapeutic use of IR. Therefore, this study investigated the possible role of ROS in IR-induced nuclei damage. The cellular components involved in the IR-induced generation of ROS were also determined, and tested for their roles in nuclei damage.

\section{Materials and methods}

Cell culture, transfection, and treatment. Jurkat T cells were grown in RPMI-1640 medium supplemented with $10 \%$ fetal bovine serum and gentamicine $(50 \mu \mathrm{g} / \mathrm{ml})$. A specific sequence of 19 nucleotides (CCAGGATTGAAGTGGATGG, residues 1130 to 1148 ) of the human Nox 1 cDNA was selected to synthesize small interfering RNAs (siRNAs) (24). The universal control sequence (AGTTCAACGACCAGTAGTC), which has no significant homology to any known mammalian genome, was used as a negative control (25). These oligonucleotides were cloned into the pSUPER vector (Oligoengine, Seattle, WA) according to the manufacturer's protocol. The vectors cloned were then introduced into the cells by electroporation. The transfected cells were selected using G418 sulfate $(4 \mathrm{mg} / \mathrm{ml})$. To treat the cells/transfectants, they were plated at a concentration of $3 \times 10^{5} \mathrm{cells} / \mathrm{ml}$, and then exposed to $\gamma$-rays from a ${ }^{137} \mathrm{Cs} \gamma$-ray source (Atomic Energy of Canada, Mississauga, Canada) with a dose rate of $3.81 \mathrm{~Gy} / \mathrm{min}$. Where specified, catalase, LY294002, and SP600125 were added at the indicated concentrations.

Analysis of cellular ROS levels. The treated and untreated control cells were exposed to $50 \mu \mathrm{M} 2^{\prime}, 7^{\prime}$-dichlorofluorescein diacetate (DCFH-DA, Molecular Probes, Eugene, OR) for 5 min, and cell-associated levels of DCF fluorescence were analyzed by flow cytometry (26).

Micronucleus assay. After $24 \mathrm{~h}$ of irradiation, the cells received cytochalasin $\mathrm{B}(3 \mu \mathrm{g} / \mathrm{ml})$ to inhibit cytokinesis, and were incubated for a further $29 \mathrm{~h}$. The cells were harvested onto glass slides using a cytocentrifuge, air-dried, and fixed with methanol:glacial acetic acid $(3: 1 \mathrm{v} / \mathrm{v})$. The slides were then stained with a Giemsa solution, and analyzed under a light microscope at a magnification of $x 1,000$. The micronuclei were scored when their diameter was $<1 / 3$ of the diameter of the main nucleus. The number of micronuclei in 300 binucleated cells was determined.

Reverse transcriptase-polymerase chain reaction (RT-PCR). Total RNA was extracted using TRIzol (Sigma, St. Louis, $\mathrm{MO})$. RT reaction was performed using total RNA as a template and a RT-for-PCR kit (Promega, Madison, WI). PCR amplification was carried out with the following primers:
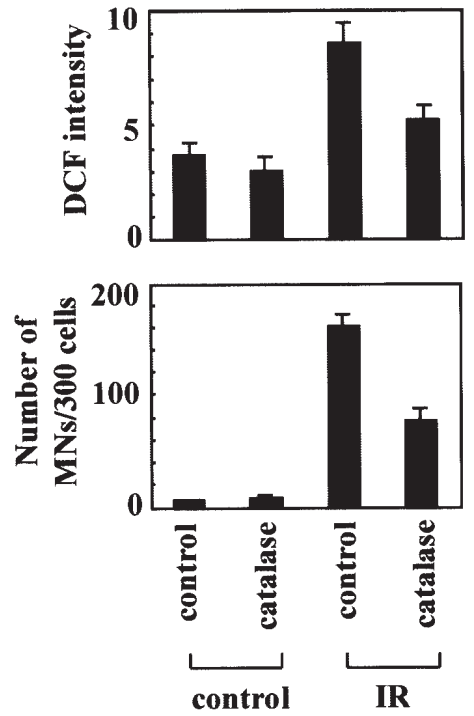

Figure 1. ROS mediate the IR-induced formation of the micronucleus. Jurkat cells were irradiated with $\gamma$-rays $(2.5 \mathrm{~Gy})$ in the presence or absence of catalase $(1,000 \mathrm{U} / \mathrm{ml})$. After $24 \mathrm{~h}$ of incubation, the cellular ROS levels were analyzed using DCF fluorescence (top panel). Alternatively, the number of micronuclei in the treated and untreated control cells was analyzed using a cytokinesis-block method (bottom panel). The values shown are the means of triplicate experiments. The error bars represent the standard deviations.

Nox1, 5'-ctgggtggttaaccactggtt-3' and 5'-accaatgccgtgaatccc taag-3'; ß-actin, 5'-gcccagagcaagagaggcat-3' and 5'-ggccatctc ttgctcgaagt- $3^{\prime}$.

Western blot analysis. The cell were lysed in Tris- $\mathrm{HCl}(40 \mathrm{mM}$, $\mathrm{pH} 8.0), 120 \mathrm{mM} \mathrm{NaCl}, 0.5 \%$ Nonidet P-40, and protease inhibitors $(2 \mu \mathrm{g} / \mathrm{ml}$ aprotinin, $2 \mu \mathrm{g} / \mathrm{ml}$ leupeptin, and $100 \mu \mathrm{g} /$ $\mathrm{ml}$ PMSF). Equal amounts of the proteins were separated by SDS-PAGE, then electrotransferred to the Immobilon membranes (Millipore, Bedford, MA), which were subsequently blotted using the indicated antibodies and visualized by chemiluminescence (ECL; Amersham Pharmacia, Uppsala, Sweden).

Phosphoinositide 3-kinase (PI3K) assay. Cells were lysed as described previously (27). Equal amounts of the lysate proteins $(400 \mu \mathrm{g})$ were immunoprecipitated with an antibody specific to the p85 subunit of PI3K (Upstate Biotechnology, Lake Placid, $\mathrm{NY}$ ). The immune complexes were washed, and resolved in 20 mM HEPES (pH 7.4), 5 mM $\mathrm{MnCl}_{2}, 10$ (M ATP, $10 \mu \mathrm{Ci}$ $\gamma$-[32P]ATP, and $2.5 \mathrm{mM}$ EGTA. L- $\alpha$-phosphatidylinositol (Sigma) $(5 \mathrm{mg} / \mathrm{ml})$ was used to initiate the kinase reactions. After 20 min of incubation, the reactions were quenched by adding $1 \mathrm{M} \mathrm{HCl}$. The phospholipids were extracted using a 1:1 mixture of chloroform and methanol, and separated by thin-layer chromatography.

c-Jun N-terminal kinase (JNK) assay. Cells were lysed in a buffer defined previously (28). The lysate proteins $(400 \mu \mathrm{g})$ were immunoprecipitated using anti-JNK (Santa Cruz Biotechnology, Santa Cruz, CA), and were resolved in $20 \mu \mathrm{l}$ of a kinase buffer containing $20 \mathrm{mM}$ Hepes ( $\mathrm{pH}$ 7.4), $10 \mathrm{mM}$ $\mathrm{MgCl}_{2}, 20 \mathrm{mM}$ B-glycerophosphate, $10 \mathrm{mM} \mathrm{NaF}, 1 \mathrm{mM}$ DTT, $0.5 \mathrm{mM}$ sodium orthovanadate, $50 \mu \mathrm{M}$ ATP, and $10 \mu \mathrm{Ci}$ 


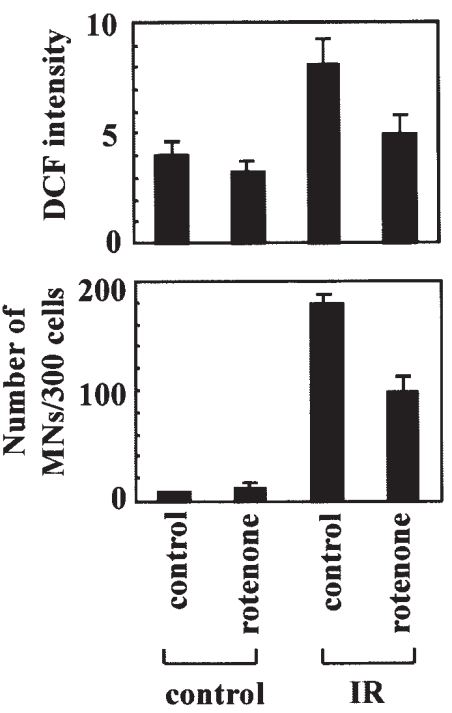

Figure 2. Role of the mitochondria in the IR-induced formation of micronuclei. Jurkat cells were irradiated with $\gamma$-rays $(2.5 \mathrm{~Gy})$ with or without rotenone $(25 \mathrm{nM})$. The cellular ROS levels (top panel) and micronucleus formation (bottom panel) were analyzed $24 \mathrm{~h}$ after irradiation.

$\gamma-\left[{ }^{32} \mathrm{P}\right] \mathrm{ATP}$. The kinase reactions were initiated by adding $2 \mu \mathrm{g}$ of the recombinant c-Jun protein (New England Biolabs, Beverly, MA). After $30 \mathrm{~min}$ of incubation, the reactions were quenched by adding a boiled sample buffer, and the proteins were subsequently separated by $12 \%$ SDS-PAGE. A PhosphoImager using Tina 2.0 software was used to analyze the results.

\section{Results}

Role of ROS in IR-induced micronuclei formation. To determine an optimal condition to analyze the role of ROS in IR-induced nuclei damage, Jurkat cells were irradiated with various doses of $\gamma$-rays. After $48 \mathrm{~h}$ of irradiation, the cellular viability was determined using a propidium iodide exclusion assay (29). The cells (>90\%) were viable when they were irradiated with up to $2.5 \mathrm{~Gy}$. However, $\gamma$-ray doses 5 Gy and higher induced cell death (data not shown). Therefore, the cells were irradiated with $2.5 \mathrm{~Gy}$, incubated for $24 \mathrm{~h}$, and their ROS levels were analyzed using DCF fluorescence. Irradiation with 2.5 Gy significantly increased the cellular ROS levels (Fig. 1, top panel). To determine if the treatment also induced nuclei damage, the irradiated cells were analyzed for the presence of micronuclei using a cytokinesis-block method. An analysis of 300 cells revealed $<10$ micronuclei in the case of the untreated control group. However, the number of micronuclei increased to $150-170$ when the cells were irradiated with 2.5 Gy (Fig. 1, bottom panel). Given the co-induction of ROS and micronuclei, it appears likely that ROS mediated the IR-induced formation of micronuclei. To investigate this possibility, the cells were irradiated in the presence of catalase, a $\mathrm{H}_{2} \mathrm{O}_{2}$ degrading enzyme. Catalase almost completely abolished the IR-induced accumulation of ROS (Fig. 1, top panel), and effectively reduced the IR-induced formation of micronuclei (Fig. 1, bottom panel). Therefore, ROS appear to be involved in the IR-induced formation of micronuclei.
(A)

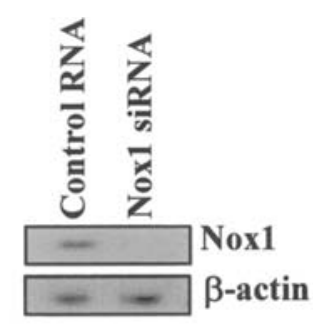

(B)

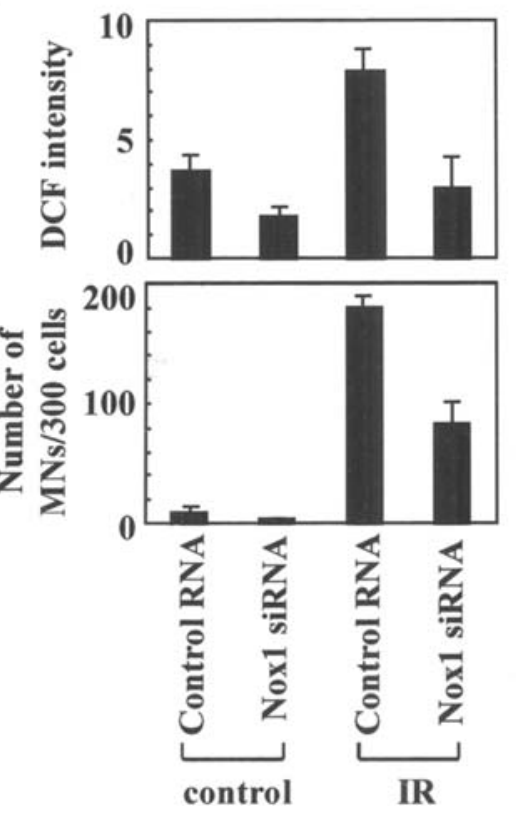

Figure 3. Role of Nox1 in the IR-induced formation of micronuclei. (A) Jurkat cells were transfected with the pSUPER vectors encoding either a nonspecific control RNA or siRNA specific for Nox1. The Nox1 levels in these transfectants were compared by RT-PCR using B-actin as the loading control. (B) The transfectants were irradiated with $\gamma$-rays $(2.5 \mathrm{~Gy})$, and the cellular ROS levels (top panel) and micronucleus formation (bottom panel) were analyzed $24 \mathrm{~h}$ after irradiation.

Role of mitochondria and Noxl in IR-induced micronuclei formation. The mitochondria have been implicated in the IRinduced generation of ROS under other experimental conditions (23). In order to confirm this under the present experimental conditions, the cells were irradiated at $2.5 \mathrm{~Gy}$ in the presence of rotenone, an inhibitor of the mitochondrial respiratory chain. The addition of rotenone efficiently suppressed the ability of IR to induce both the accumulation of ROS (Fig. 2, top panel) and the formation of micronuclei (Fig. 2, bottom panel). This suggests that IR promotes the mitochondrial production of ROS, which then contributes to the formation of micronuclei.

Recent studies suggest that Nox1, a member of the Nox family of NADPH oxidase, is expressed in nonphagocytic cells, and responds to cell stimulation with growth factors by generating ROS (22). RT-PCR confirmed that Nox 1 is expressed in Jurkat cells (Fig. 3A). A disruption of Nox1 expression by RNA interference attenuated the IR-induced accumulation of ROS and formation of micronuclei (Fig. 3B). Therefore, Nox 1 appears to be an additional source of ROS that can damage the nuclei in this system. 
(A)

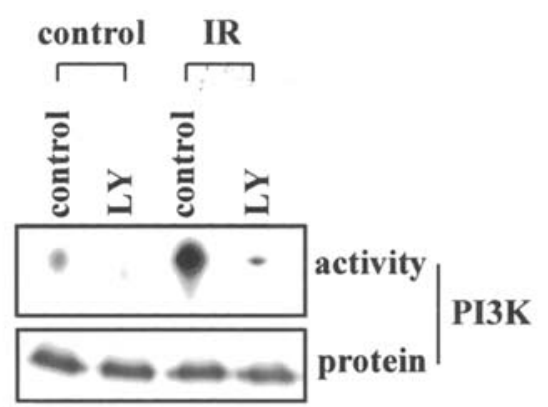

(B)
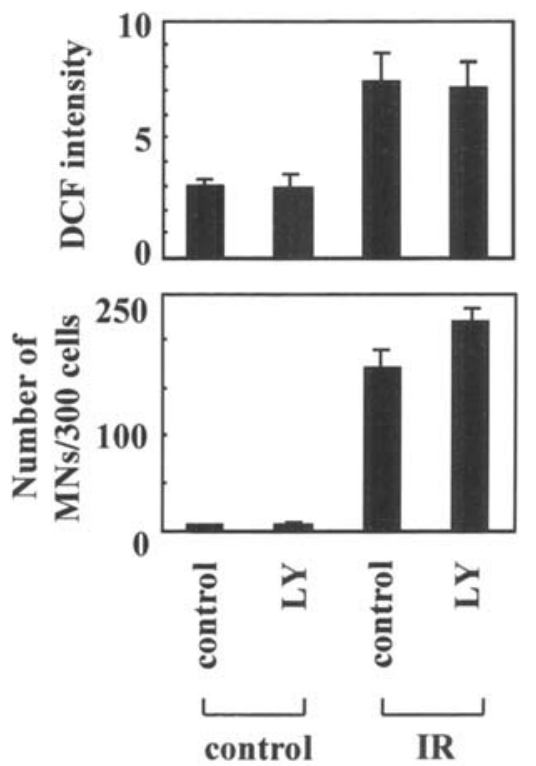

Figure 4. PI3K is not involved in the IR-induced formation of micronuclei. (A) Jurkat cells were irradiated with $\gamma$-rays $(2.5 \mathrm{~Gy})$ in the presence or absence of LY294002 $(5 \mu \mathrm{M})$. At the end of $15 \mathrm{~min}$ of incubation, the cells were lysed, and PI3K was immunoprecipitated with anti-p85. The PI3K activities in precipitates were analyzed using $\mathrm{L}$ - $\alpha$-phosphatidylinositol as a substrate (top panel). The levels of its $\mathrm{p} 85$ subunit in samples were compared by Western blotting with anti-p85 (bottom panel). (B) The incubation was extended for up to $24 \mathrm{~h}$, and the cellular ROS levels (top panel) and micronucleus formation (bottom panel) were analyzed.

PI3K is not involved in IR-induced nucleus damage. PI3K is a signaling enzyme that is involved in the generation of ROS induced by chemokines (30), growth factors (22), and serum withdrawal (27). The potential role of PI3K in IR-induced ROS accumulation was examined by analyzing the irradiated cells for their PI3K activity using an in vitro kinase assay. As shown in Fig. 4A, 2.5 Gy of IR induced the activation of PI3K. This event was abolished when cells were irradiated in the presence of LY294002, a PI3K inhibitor. However, LY294002 failed to significantly attenuate the IR-induced accumulation of ROS and the formation of micronuclei (Fig. 4B). Therefore, these events appear to occur independently of PI3K.

Role of JNK in IR-induced ROS accumulation and micronucleus formation. Given that JNK can mediate the production of ROS under certain experimental conditions $(31,32)$, this study examined this role for JNK in the present system. An in vitro kinase assay revealed that IR activated JNK in Jurkat cells (Fig. 5A). This was prevented in the presence of
(A)

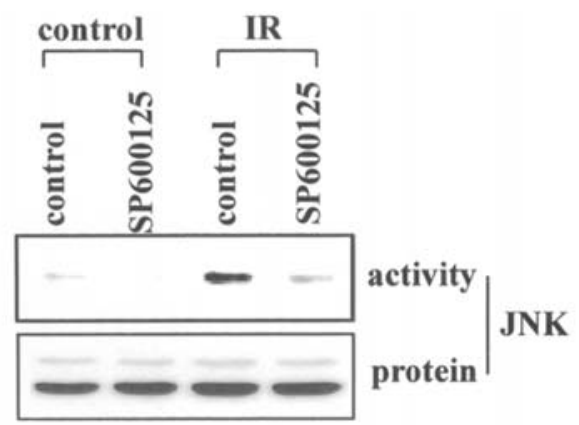

(B)
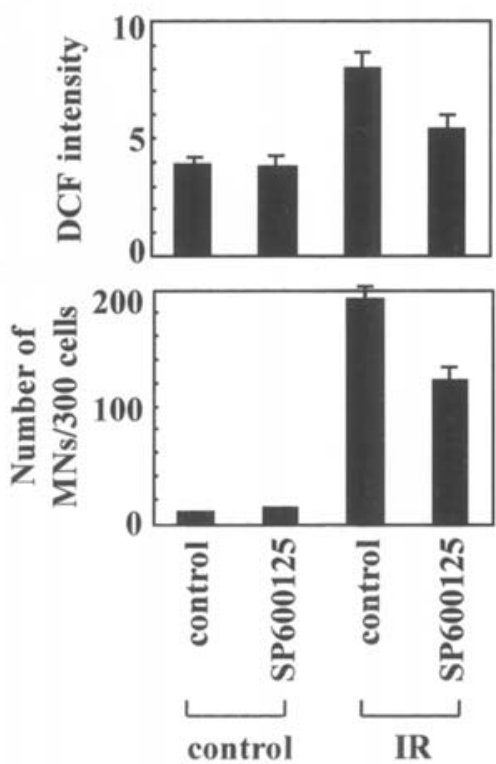

Figure 5. JNK mediates the IR-induced formation of micronuclei. (A) The Jurkat cells were irradiated with $\gamma$-rays $(2.5 \mathrm{~Gy})$ in the presence or absence of SP600125 $(2.5 \mu \mathrm{M})$. After $3 \mathrm{~h}$ of incubation, the JNK activity was analyzed by an in vitro kinase assay. (B) The incubation was extended for up to $24 \mathrm{~h}$, and the cellular ROS levels (top panel) and micronucleus formation (bottom panel) were analyzed.

SP600125, an inhibitor of JNK, which resulted in a decrease in the IR-induced accumulation of ROS and the formation of micronucleus (Fig. 5B). Therefore, JNK appears to mediate the ability of IR to induce ROS and micronucleus formation.

The finding that JNK acts as an upstream inducer of ROS contrasts with a previous study showing that ROS mediate the activation of JNK under various experimental conditions $(10,28)$. To confirm this latter event in the irradiated cells, the Jurkat cells were irradiated in the presence or absence of catalase and rotenone. The addition of either of these attenuated the IR-induced activation of JNK (Fig. 6). Similar results were obtained when the Nox1 levels were reduced by RNA interference. These observations suggest that the ROS induced by the mitochondria and Nox1 are essential for the IR-induced activation of JNK. Overall, it appears that ROS and JNK mutually interact to enhance the level/activity of the other in irradiated cells.

\section{Discussion}

The present study showed that ROS act as mediators of IRinduced micronucleus formation. This was initially suggested 


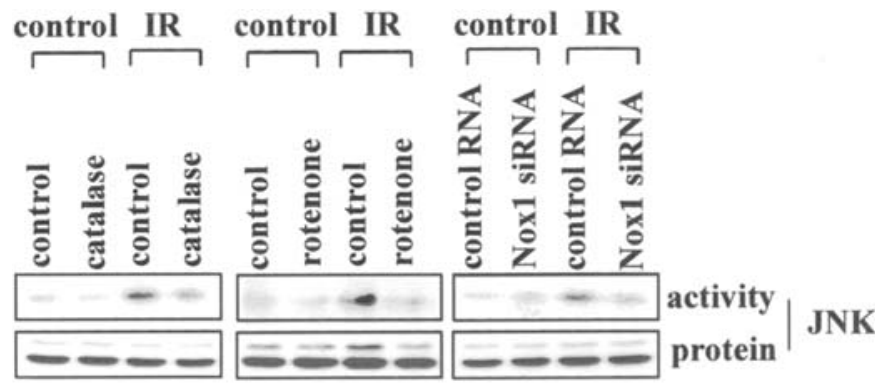

Figure 6. ROS mediate IR-induced JNK activation. Untransfected Jurkat cells or indicated transfectants were irradiated with $\gamma$-rays $(2.5 \mathrm{~Gy})$. Where indicated, catalase $(1,000 \mathrm{U} / \mathrm{ml})$ and rotenone $(25 \mathrm{nM})$ were added. The JNK activity was analyzed by an in vitro kinase assay $3 \mathrm{~h}$ after irradiation.

by the observation that the IR-induced accumulation of ROS is accompanied by the formation of micronuclei, and was more directly supported by the finding that the elimination of the IR-induced ROS using catalase reduced the formation of micronuclei. Although the role of ROS in IR-induced genotoxic damage has been proposed at the levels of DNA and chromosomes $(6,7)$, this is the first study to show that ROS are also involved in the gross nuclei damage.

Despite the well-recognized ability of IR to induce the generation of $\operatorname{ROS}(6,8,23)$, the cellular processes leading to this event are not completely understood. Under the present experimental conditions, the IR-induced accumulation of ROS was attenuated either when rotenone was added to the culture or when the cellular Nox1 levels were reduced by RNA interference. This suggests that IR can stimulate both the mitochondria and Nox1 to produce ROS in a single cell type. Neither rotenone nor Nox1 siRNA reduced the IR-induced formation of micronucleus, suggesting that the ROS involved in this process are generated, at least in part, by the mitochondria and Nox1. While IR has long been considered to possess high energy that can attack the cellular components directly, these results suggest that IR can destroy the nuclei through biological processes.

An additional finding of this study is that cellular signaling molecules can also participate in the process leading to the IR-induced damage to the nucleus. Given that JNK $(31,32)$ and PI3K $(22,27,30)$ can mediate the induction of ROS under other experimental conditions, this study investigated their roles in this system and found that JNK is involved in the IRinduced generation of ROS and micronucleus formation. IR activated JNK in Jurkat cells, and the prevention of this event using its specific inhibitor SP600125 attenuated the ability of IR to induce the accumulation of ROS and formation of micronuclei. These effects were not observed using LY294002, a PI3K inhibitor, although IR induced the activation of PI3K in Jurkat cells. Therefore, PI3K does not appear to be involved in the accumulation of ROS induced under these experimental conditions. It is unclear how JNK induces the generation of ROS in this system. The IR-activated JNK might stimulate the mitochondria, Nox1, or an alternative unidentified source of ROS. We are currently attempting to establish biochemical markers for the mitochondria and Nox 1 stimulation to directly assess this possibility.
These results suggest that IR activates JNK in a ROSdependent manner. This is consistent with the ability of ROS to induce the activation of JNK under various experimental settings $(10,28)$, and is supported by the observation that catalase blocks the IR-induced activation of JNK in this system. This effect was also observed using rotenone or Nox1 siRNA. Therefore, the ROS generated by both the mitochondria and Nox1 appear to be involved in the IR-induced activation of JNK. The finding that JNK and ROS enhance the level/activity of each component in a single model, suggests their positive mutual interaction in a way that either ROS or JNK responds to IR to enhance the level/activity of the other. This in turn elevates that of the initiator component. In this regard, JNK might not simply mediate the ability of IR to induce ROS but amplify the ROS-inducing process.

In summary, this study showed that ROS contribute to the IR-induced formation of micronuclei. The cellular components involved in this process were also determined. These findings significantly advance our understandings of how IR induces genetic instability.

\section{Acknowledgements}

This study was supported by Korea Science and Engineering Foundation (KOSEF) and Ministry of Science and Technology (MOST), Korean government, through its National Nuclear Technology Program.

\section{References}

1. Chadwick KH and Leenhouts HP: Comments on the paper: modelling radiation-induced chromosome aberrations. Int $\mathrm{J}$ Radiat Biol 79: 287-288, 2003.

2. Huang L, Snyder AR and Morgan WF: Radiation-induced genomic instability and its implications for radiation carcinogenesis. Oncogene 22: 5848-5854, 2003.

3. Morgan WF, Hartmann A, Limoli CL, Nagar S and Ponnaiya B: Bystander effects in radiation-induced genomic instability. Mutat Res 504: 91-100, 2002.

4. Baverstock KF: Abnormal distribution of double strand breaks in DNA after direct action of ionizing energy. Int $\mathrm{J}$ Radiat Biol Relat Stud Phys Chem Med 47: 369-374, 1985.

5. Siddiqi MA and Bothe E: Single- and double-strand break formation in DNA irradiated in aqueous solution: dependence on dose and $\mathrm{OH}$ radical scavenger concentration. Radiat Res 112: 449-463, 1987.

6. Morales A, Miranda M, Sanchez-Reyes A, Biete A and Fernandez-Checa JC: Oxidative damage of mitochondrial and nuclear DNA induced by ionizing radiation in human hepatoblastoma cells. Int J Radiat Oncol Biol Phys 42: 191-203, 1998.

7. Ogawa Y, Kobayashi T, Nishioka A, Kariya S, Hamasato S, Seguchi $\mathrm{H}$ and Yoshida S: Radiation-induced reactive oxygen species formation prior to oxidative DNA damage in human peripheral T cells. Int J Mol Med 11: 149-152, 2003.

8. Tominaga H, Kodama S, Matsuda N, Suzuki K and Watanabe M: Involvement of reactive oxygen species (ROS) in the induction of genetic instability by radiation. J Radiat Res (Tokyo) 45: 181-188, 2004.

9. Lee BR and Um HD: Hydrogen peroxide suppresses U937 cell death by two different mechanisms depending on its concentration. Exp Cell Res 248: 430-438, 1999.

10. Martindale JL and Holbrook NJ: Cellular response to oxidative stress: signaling for suicide and survival. J Cell Physiol 192: 1-15, 2002.

11. Um HD, Orenstein JM and Wahl SM: Fas mediates apoptosis in human monocytes by a reactive oxygen intermediate dependent pathway. J Immunol 156: 3469-3477, 1996.

12. Um HD, Cho YH, Kim do K, Shin JR, Lee YJ, Choi KS, Kang JM and Lee MG: TNF-alpha suppresses dendritic cell death and the production of reactive oxygen intermediates induced by plasma withdrawal. Exp Dermatol 13: 282-288, 2004. 
13. Corda S, Laplace C, Vicaut E and Duranteau J: Rapid reactive oxygen species production by mitochondria in endothelial cells exposed to tumor necrosis factor-alpha is mediated by ceramide. Am J Respir Cell Mol Biol 24: 762-768, 2001.

14. Panduri V, Weitzman SA, Chandel NS and Kamp DW: Mitochondrial-derived free radicals mediate asbestos-induced alveolar epithelial cell apoptosis. Am J Physiol Lung Cell Mol Physiol 286: L1220-L1227, 2004.

15. Yoon YS, Lee JH, Hwang SC, Choi KS and Yoon G: TGF beta1 induces prolonged mitochondrial ROS generation through decreased complex IV activity with senescent arrest in Mv1Lu cells. Oncogene 24: 1895-1903, 2005.

16. Brandes RP and Kreuzer J: Vascular NADPH oxidase: molecular mechanisms of activation. Cardiovasc Res 65: 16-27, 2005.

17. El Bekay R, Alvarez M, Monteseirin J, Alba G, Chacon P, Vega A, Martin-Nieto J, Jimenez J, Pintado E, Bedoya FJ and Sobrino F: Oxidative stress is a critical mediator of the angiotensin II signal in human neutrophils: involvement of mitogenactivated protein kinase, calcineurin, and the transcription factor NF-kappaB. Blood 102: 662-671, 2003.

18. Forman HJ and Torres M: Reactive oxygen species and cell signaling: respiratory burst in macrophage signaling. Am J Respir Crit Care Med 166: S4-S8, 2002.

19. Hordijk PL: Regulation of NADPH oxidases: the role of Rac proteins. Circ Res 98: 453-462, 2006.

20. Lambeth JD: NOX enzymes and the biology of reactive oxygen. Nat Rev Immunol 4: 181-189, 2004.

21. Sumimoto H, Miyano K and Takeya R: Molecular composition and regulation of the Nox family NAD $(\mathrm{P}) \mathrm{H}$ oxidases. Biochem Biophys Res Commun 338: 677-686, 2005.

22. Park HS, Lee SH, Park D, Lee JS, Ryu SH, Lee WJ, Rhee SG and Bae YS: Sequential activation of phosphatidylinositol 3-kinase, beta Pix, Rac1, and Nox1 in growth factor-induced production of $\mathrm{H}_{2} \mathrm{O}_{2}$. Mol Cell Biol 24: 4384-4394, 2004.

23. Lee YJ, Lee DH, Cho CK, Chung HY, Bae S, Jhon GJ, Soh JW, Jeoung DI, Lee SJ and Lee YS: HSP25 inhibits radiationinduced apoptosis through reduction of PKCdelta-mediated ROS production. Oncogene 24: 3715-3725, 2005.
24. Brummelkamp TR, Bernards R and Agami R: A system for stable expression of short interfering RNAs in mammalian cells. Science 296: 550-553, 2002.

25. Yu L, Wu G, Wang L, Wang $\mathrm{H}$ and Zhang G: Transient reduction of PTI-1 expression by short interfering RNAs inhibits the growth of human prostate cancer cell lines. Tohoku J Exp Med 209: 141-148, 2006.

26. Bass DA, Parce JW, Dechatelet LR, Szejda P, Seeds MC and Thomas M: Flow cytometric studies of oxidative product formation by neutrophils: a graded response to membrane stimulation. J Immunol 130: 1910-1917, 1983.

27. Lee SB, Cho ES, Yang HS, Kim HG and Um HD: Serum withdrawal kills U937 cells by inducing a positive mutual interaction between reactive oxygen species and phosphoinositide 3-kinase. Cell Signal 17: 197-204, 2005.

28. Kim DK, Cho ES, Seong JK and Um HD: Adaptive concentrations of hydrogen peroxide suppress cell death by blocking the activation of SAPK/JNK pathway. J Cell Sci 114: 4329-4334, 2001.

29. Mangan DF, Welch GR and Wahl SM: Lipopolysaccharide, tumor necrosis factor-alpha, and IL-1 beta prevent programmed cell death (apoptosis) in human peripheral blood monocytes. $\mathrm{J}$ Immunol 146: 1541-1546, 1991.

30. Sasaki T, Irie-Sasaki J, Jones RG, Oliveira-dos-Santos AJ, Stanford WL, Bolon B, Wakeham A, Ltie A, Bouchard D, Kozieradzki I, Joza N, Mak TW, Ohashi PS, Suzuki A and Penninger JM: Function of PI3Kgamma in thymocyte development, T cell activation, and neutrophil migration. Science 287: 1040-1046, 2000.

31. Antosiewicz J, Herman-Antosiewicz A, Marynowski SW and Singh SV: c-Jun NH(2)-terminal kinase signaling axis regulates diallyl trisulfide-induced generation of reactive oxygen species and cell cycle arrest in human prostate cancer cells. Cancer Res 66: 5379-5386, 2006.

32. Ventura JJ, Cogswell P, Flavell RA, Baldwin AS Jr and Davis RJ: JNK potentiates TNF-stimulated necrosis by increasing the production of cytotoxic reactive oxygen species. Genes Dev 18: 2905-2915, 2004. 